\title{
BMJ Open Effectiveness of intraoperative peritoneal lavage (IOPL) with saline in patient with intra-abdominal infections: a systematic review and meta-analysis protocol
}

\author{
Qi Zhou, ${ }^{1,2,3,4}$ Qianling Shi, ${ }^{1,5}$ Xuan Yu (1) , ${ }^{2,3,4}$ Zijun Wang, ${ }^{2,3,4}$ Jingyi Zhang, ${ }^{6}$ \\ Nan Yang, ${ }^{2,3,4}$ Jianjian Wang, ${ }^{6}$ Yanfang Ma (10 , ${ }^{2}$ Xufei Luo, ${ }^{6}$ Yangqin Xun, ${ }^{2,3,4}$ \\ Siya Zhao, ${ }^{6}$ Bobo Zheng, ${ }^{7}$ Wenbo Meng (D) , ${ }^{8}$ Kehu Yang (i) , ${ }^{1,2,3,4}$ \\ Yaolong Chen (D) , 2,3,4 Robert Sawyer ${ }^{9,10}$
}

To cite: Zhou Q, Shi Q, Yu X, et al. Effectiveness of intraoperative peritoneal lavage (IOPL) with saline in patient with intra-abdominal infections: a systematic review and metaanalysis protocol. BMJ Open 2020;10:e036273. doi:10.1136/ bmjopen-2019-036273

- Prepublication history and additional material for this paper are available online. To view these files, please visit the journal online (http://dx.doi. org/10.1136/bmjopen-2019036273).

QZ and QS contributed equally.

Received 08 December 2019

Revised 17 March 2020

Accepted 13 May 2020

Check for updates

(C) Author(s) (or their employer(s)) 2020. Re-use permitted under CC BY-NC. No commercial re-use. See rights and permissions. Published by BMJ.

For numbered affiliations see end of article.

Correspondence to

Dr Yaolong Chen;

chenyaolong@|zu.edu.cn and

Professor Kehu Yang;

kehuyangebm2006@126.com

\section{ABSTRACT}

Introduction Intra-abdominal infections (IAls) are common surgical emergencies and cause a significant worldwide burden per year. Since the concept of intraoperative peritoneal lavage (IOPL) was proposed in 1905 , it has been widely used in the surgery practice. However, the effectiveness of IOPL in patients with IAls has always been controversial. Our objective is to identify whether it is beneficial to flush the abdominal cavity with saline in IAls surgery through a comprehensive systematic review and meta-analysis.

Methods and analysis This protocol is reported in line with the Preferred Reporting Items for Systematic Review and Meta-Analysis Protocols. Electronic databases (including the Cochrane library, MEDLINE, EMBASE, Web of Science, etc) and clinical trial registry platforms will be searched from inception to 8 September 2019. Randomised controlled trials, quasi-randomised clinical trials and cohort studies comparing IOPL and suction alone in IAls will be included. The primary outcomes are mortality and abscess rate. Two independent reviewers will screen literature, collect data and assess risk of bias of included studies. Discussion or a third reviewer will be referred for any disagreements. The Grading of Recommendations Assessment, Development and Evaluation approach will be used to assess the quality of the evidence. We will perform meta-analysis using randomeffects model. Subgroup analysis, sensitivity analysis and publication bias will be conducted if data are enough. Ethics and dissemination Ethical approval is not required for this systematic review and meta-analysis protocol. Results of this study will be published in a peerreviewed journal, presented at relevant conferences and disseminated to local and international policy makers. PROSPERO rregistration number CRD42019145109.

\section{INTRODUCTION}

Intra-abdominal infections (IAIs) are a series of infectious diseases caused by the invasion of bacteria into the abdominal cavity and organs, which representing with a wide spectrum of pathological conditions, ${ }^{1}$ of which, appendicitis is one of the most common symptoms
Strengths and limitations of this study

- This systematic review will be the first to evaluate effectiveness of intraoperative peritoneal lavage with saline in patients with intra-abdominal infections.

- Studies will be obtained from a wide range sources including electronic databases, clinical trial registry platforms, Google Scholar, reference lists of relevant reviews and grey literature.

- This systematic review protocol has been designed according to the Preferred Reporting Items for Systematic Review and Meta-Analysis Protocols.

- Methodological biases in the included primary studies may cause uncertainty in the results of the present study.

with the world prevalence of $7 \%-8 \% .^{2-4}$ In some cases, infection will extend beyond the organs and cause either localised or diffuse peritonitis; then it develops into complicated IAIs (cIAIs), leading to sepsis, multiple organ dysfunction syndrome and even death. ${ }^{1}$ The mortality rate of IAIs varies with the infection location, pathogen type and inflammation extension. ${ }^{56} \mathrm{~A}$ multicentre study of 68 medical institutions showed the overall mortality of cIAIs was $10.5 \% .^{7}$ While another multicentre study of 132 medical institutions indicated that the progression to septic shock would significantly increase the fatality rate of cIAIs from $1.2 \%$ to $67.8 \% .^{2}$ In line with this, more and more institutions have developed guidelines to manage IAIs, such as the World Society of Emergency Surgery, ${ }^{89}$ the Surgical Infection Society ${ }^{10}$ and the French Society of Anesthesia and Resuscitation. ${ }^{11}$

In general, principles of effective treatment in IAIs include early recognition, adequate source control and appropriate antimicrobial therapy, ${ }^{8}$ of which, source control is the core. 
Since the concept of intraoperative peritoneal lavage (IOPL) was first proposed by Price,${ }^{12}$ its clinical effectiveness on patients with IAIs has been investigated by some authors; however, it still be a controversial issue. Some authors argued that peritoneal irrigation would increase the risk of abscess rate and may just be a ritual that with no biological advantages, but others argued that dilution is the solution of the pollution. ${ }^{1314}$ Published systematic reviews only focused on the complex appendicitis instead of addressing problems of IAIs, and they did not account for the major outcomes. ${ }^{15} 16$ Thus, with the limited evidence from the literature, there is no clear consensus. Moreover, until now, published clinical practice guidelines are not sure whether it is necessary to irrigate the source of infection during the IAIs surgery. ${ }^{8-11}$

Therefore, the purpose of this study is to perform a comprehensive systematic review and meta-analysis to explore whether it is beneficial to flush the abdominal cavity with saline in IAIs surgery, and finally provide evidence support for IAIs guidelines.

\section{OBJECTIVE}

The primary objective of this systematic review is to synthesise the current evidence on the effectiveness of IOPL in patients with IAIs and to provide evidence support for clinical practice guidelines and clinical decision making.

\section{METHODS}

Protocol for this systematic review and meta-analysis was prepared complying with the Centre of Reviews and Dissemination guidelines ${ }^{17}$ and reported according to the Preferred Reporting Items for Systematic Review and Meta-Analysis Protocols. ${ }^{18}$

\section{Eligibility criteria}

\section{Study characteristics}

This systematic review will include randomised controlled trials (RCTs), quasi-randomised clinical trials and cohort studies which comparing IOPL and suction alone. Also, these studies should report data of major clinical outcomes and details of irrigation. Duplicates and fulltext unavailable due to specific reasons will be excluded. Studies with specific data missing will also be excluded.

\section{Participants}

Patients diagnosed with IAIs will be included, without restrictions on age, race, gender and geographical location or setting.

\section{Interventions}

This systematic review will include studies which comparing IOPL with suction alone, and the used irrigation can be low, warm and normal temperature saline or Ringer's solution. Studies of all patients received the same antibiotic therapy postoperatively or preoperatively will be included. In this systematic review, we will exclude trials if patients in the intervention group received irrigation mixed with antibiotics or combined with drainage.

\section{Outcomes}

The primary outcomes are mortality and abscess rate. The secondary outcomes include wound infection, postoperative complications, postoperative duration of stay, operative time, hospital charges, reoperation and readmission. In terms of postoperative complications' incidence, it not only includes infectious complications such as abscess rate and wound infection, but non-infectious complications such as bowel obstruction also will be included.

\section{Search methods for study identification}

\section{Search approach}

A set of search terms will be drawn up by an experienced librarian, and a comprehensive search will be independently performed by two reviewers from inception to 8 September 2019 in the following electronic databases: the Cochrane library, MEDLINE (via PubMed), EMBASE, Web of Science, CBM (China Biology Medicine disc), CNKI (China National Knowledge Infrastructure) and Wanfang Data. ${ }^{19}$ There will be no language and publication status restrictions. We will also search clinical trial registry platforms (the WHO Clinical Trials Registry Platform (http://www.who.int/ictrp/en/), US National Institutes of Health Trials Register (https://clinicaltrials. gov/)), Google Scholar (https://scholar.google.nl/) and reference lists of relevant reviews and grey literature for unpublished or further potential studies. Detailed inclusion and exclusion criteria are presented in table 1.

\section{Search strategy}

In order to achieve the most inclusive search, the search strategy will be peer reviewed by a second information specialist. Our search strategy will be performed using the medical subject heading terms and text words combined in a Boolean search. More strategy details can be found in the online supplementary file 1 .

\section{Selection of studies}

After eliminating duplicates, two reviewers will independently perform searches by two steps, any discrepancies will be settled by discussion or consulting a third reviewer. The specific bibliographic software EndNote will be used. Prior to the formal selection, a pilot of 50 random sample citations will be conducted until sufficient agreement will be reached.

In step 1, all titles and abstracts will be screened using the predefined criteria, studies will be subcategorised into three (included, excluded and unsure) groups. For articles with insufficient information, reviewers will retrieve the full-text in the next stage. In step 2, full-texts of those potentially eligible and unclear studies will be reviewed to identify the final inclusion. All the reasons for exclusion of ineligible studies will be recorded, the process of study selection will be documented using a Preferred Reporting Items for Systematic Reviews and Meta-Analyses (PRISMA) flow diagram. ${ }^{18}$ 
Table 1 Inclusion and exclusion criteria

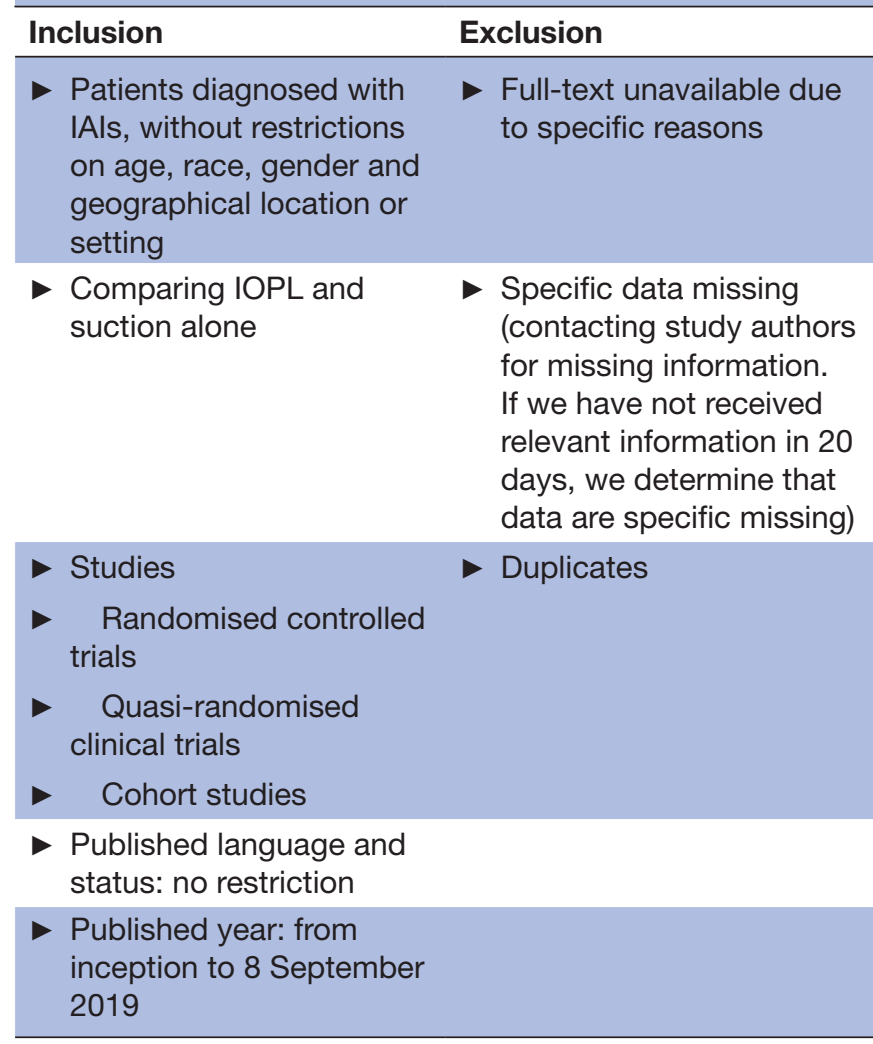

IAI, intra-abdominal infection; IOPL, intraoperative peritoneal lavage.

\section{Data extraction}

Two reviewers will extract data independently with a standard data collection form, any disagreements will be resolved by consensus, and a third reviewer will check for data's consistency and accuracy. Before the formal extraction, the form will be piloted on a random sample of five included studies and the final version will counselling with clinicians.

Data extracted will include: (1) basic information: title, first author, publication year, funding and study design; (2) participants: baseline characteristics and inclusion/ exclusion criteria of patients; (3) details of the intervention and control conditions; (4) outcomes: for dichotomous, we will abstract the number of events and total participants in per group; for continuous, we will abstract mean, SD and the number of total participants in per group. Outcomes with zero event will be reported, but theses will be excluded from analysis. For missing data or reported in an unusable way (eg, when a study is identified as abstract only or data are not available for subgroups), we will summarise them in the table and deal with them according to the strategies outlined in the data synthesis section.

\section{Risk of bias in individual studies}

For included RCTs, two reviewers will assess the risk of bias independently using Cochrane risk-of-bias tool, ${ }^{20}$ any disagreements will be discussed in a consensus meeting. It consists of seven domains: random sequence generation, allocation concealment, blinding of participants and personnel, blinding of outcome assessment, incomplete outcome data, selective outcome reporting and other bias. For each, we will grade as 'low', 'unclear' and 'high'. For those included non-RCTs, ROBINS-I tool will be used..$^{21}$ ROBINS-I is a new tool for evaluating risk of bias that studies did not use randomisation to allocate units. It consists of seven domains: bias due to confounding, bias in selection of participants, bias in classification of interventions, bias due to departures from intended interventions, bias due to missing data, bias in outcome measurement and bias in selective reporting. For each, we will grade as 'low risk', 'moderate risk', 'serious risk', 'critical risk' and 'no information'.

We will also follow the principles of the risk of bias overall assessment, using above tools to produce a 'risk of bias' summary table, which including each item, judgement and support for the judgement. The higher proportion of studies assessed as high risk, the more cautious should be used to analyse and interpret results and the grading of the quality of evidence will be lower. ${ }^{20}{ }^{21}$

\section{Quality of the evidence}

Two reviewers will assess the quality of evidence independently using the Grading of Recommendations Assessment, Development and Evaluation (GRADE). We will produce 'summary of findings' table using the GRADEpro software. ${ }^{22}{ }^{23}$ This table will include overall grading of evidence body and each prespecified outcome that will be accounted for meta-analysis.

In this approach, direct evidence from RCTs begins at high quality, while observational study begins at low; however, the overall quality will be analysed on five downgrade considerations (study limitations, consistency of effect, imprecision, indirectness and publication bias) and three up-grade considerations (large magnitude of effect, dose-response relation and plausible confounders or biases).${ }^{24-29}$ Hence, the quality of evidence will be classified as high, moderate, low and very low, which will be reflecting to what extent that we are confident the effect estimates are correct.

\section{Data synthesis}

\section{Meta-analysis}

In this systematic review, if the data are similar enough for pooling to make sense, we will consider meta-analysis by using Stata V.14 software (Stata Corp LLC) ${ }^{30}$; however, if data are too heterogeneous, a qualitative synthesis will be done in our systematic review. For dichotomous data, we will analyse risk ratios with 95\% CIs; for continuous data, we will analyse mean difference (MD) or standardised MD with 95\% CI, depending on whether the same scale was used. In terms of missing data, we will correspondence with study authors or calculate them according to the Cochrane Handbook for Systematic Reviews of Interventions. ${ }^{31}$ For example, we will calculate missing SD from $\mathrm{SE}, 95 \% \mathrm{CI}$ or $\mathrm{p}$ values, and if IQR and medians are 
provided, we will impute missing mean from the median and then calculate the SD. If none of these options are available, we will impute from other meta-analysis or through modelling if linear regression exists to find the data. When the missing data cannot be found through the above methods, the study will be excluded.

As clinical and methodological heterogeneity is expected in the study design, characteristics of participants, interventions and outcome measures, we will use random-effects model $^{32}$ and the level of statistical significance will be set at $\mathrm{p}<0.05$ with two-sided. Statistical heterogeneity will be assessed by forest plot and using the $\chi^{2}$ and $\mathrm{I}^{2}$ to test. A value of $\mathrm{I}^{2}$ from $0 \%$ to $40 \%$ represents 'not important'; $30 \%$ to $60 \%$ represents 'moderate'; $50 \%$ to $90 \%$ represents 'substantial'; $75 \%$ to $100 \%$ represents 'considerable', and heterogeneity will be defined with $\mathrm{p}<0.10$ and $\mathrm{I}^{2}>50 \%$. If we detect heterogeneity, we will perform subgroup and sensitivity analysis to explore the reasons. To avoid the publication bias, the first strategy is to perform the most inclusive search in the search stage. If sufficient number of studies (at least 10 trials) are available,${ }^{31}$ we will use funnel plots and Egger regression test to assess publication bias, and significant results $(p>0.1)$ shall suggest significance. ${ }^{33}$

\section{Subgroup analysis}

We plan to carry out the following aspects of subgroups analysis:

1. The area of IAIs. There are different pathological conditions in IAIs, including infections of single organ, peritonitis and intra-abdominal abscesses. They vary from anatomy of infected site to extension of inflammation (localised, diffused or mixed). Thus, for different IAIs, efficacy of IOPL maybe various. ${ }^{1}$

2. Type of patients. Due to the underdeveloped immune system and low drug metabolism, principles of treatment and IAIs clinical manifestations are different in children and adults. Therefore, it is essential to explore the differential impact of IOPL among children and adults.

3. Study design. In a multicentric cohort study ( $\mathrm{n}=669)$, results showed that comparing with suction alone, peritoneal irrigation was associated with a lower rate of postoperative abscess in children with perforated appendicitis. ${ }^{34}$ However, a prospective RCT $(\mathrm{n}=100)$ suggested that children with complicated appendicitis, there was no difference infectious complication rates between two groups. ${ }^{35}$ Therefore, it is necessary to carry out subgroup analysis in study design.

\section{Sensitivity analysis}

We will also plan to perform multiple sensitivity analysis to assess the robustness of our findings.

1. Excluding studies with low-quality (RCTs at high risk of bias and non-randomized control trails (non-RCTs) at critical risk of bias).

2. Excluding trials in which mean or SD, or both of them were imputed for missing data.
3. Excluding trials in which low temperature normal saline was used.

\section{REPORTING}

We will follow the PRISMA statement to report our findings. ${ }^{36}$

\section{DISCUSSION}

According to the best of our knowledge, this systematic review will be the first to investigate the clinical effectiveness of IOPL with saline in patient with IAIs. In the world wide, IAIs have caused increasing morbidity, decreasing quality of life and having a significant financial cost, which bring huge burden per year for patients, clinicians, caregivers and society. ${ }^{25-7}$ Since the concept of IOPL was first proposed in the 20th century, it has been widely used in surgical practice; however, its clinical efficacy was controversial until now. In the published guidelines, there were no consensus and existing recommendations were opposite. ${ }^{8-10}$ Previous systematic reviews only focused on the complex appendicitis and did not take the major outcomes such as mortality and reoperation into consideration. ${ }^{1516}$

Therefore, given such an urgent demand, a systematic review including a comprehensive review of literature, rigorous evidence synthesis by multiple authors and meta-analysis to obtain estimate of treatment effects for patient-important outcomes, and to guide how can we provide evidence-based treatment for IAIs in the future. To ensure the transparency of the systematic review, prospective registration and protocol are necessary. ${ }^{37} \mathrm{We}$ will ensure that our study will provide rigorous evidence regarding whether it is beneficial to flush the abdominal cavity with saline in IAIs surgery. It may also allow to identify research gaps through the literature and to definite future research goals.

\section{ETHICS AND DISSEMINATION}

There will be no research ethics required for this systematic review protocol due to no patient data being collected. Results obtained from this study will be published in a peer-reviewed journal, presented at relevant conferences and disseminated to local and international policy makers.

\section{Author affiliations}

${ }^{1}$ The First School of Clinical Medicine, Lanzhou University, Lanzhou, China ${ }^{2}$ Evidence-Based Medicine Center, School of Basic Medical Sciences, Lanzhou University, Lanzhou, China

${ }^{3}$ Lanzhou University, an Affiliate of the Cochrane China Network, Lanzhou, China ${ }^{4}$ WHO Collaborating Centre for Guideline Implementation and Knowledge Translation, Lanzhou University, Lanzhou, China

${ }^{5}$ The First Hospital of Lanzhou University, Lanzhou University, Lanzhou, China

${ }^{6}$ School of Public Health, Lanzhou University, Lanzhou, China

${ }^{7}$ Department of Gastrointestinal Surgery, West China Hospital, Sichuan University, Chengdu, China

${ }^{8}$ Department of Special Minimally Invasive Surgery, The First Hospital of Lanzhou University, Lanzhou University, Lanzhou, China 
${ }^{9}$ Department of Surgery, Western Michigan University, Kalamazoo, Michigan, USA ${ }^{10}$ College of Engineering and Applied Sciences, Western Michigan University, Kalamazoo, Michigan, USA

Contributors $\mathrm{YC}$ is the guarantor of this study. All the authors contributed to the conception and design of the protocol as follows. RS, YC and KY developed the review question and planned for the review. $\mathrm{XY}$ was leading the protocol development and dissemination, QZ and QS worked on the topic refinement, designed the search strategy and selection forms. ZW, YX, XL and SZ contributed to the topic of review design and will carry out the literature search. JZ, NY, JW, YM will assess risk of bias. WM and BZ provided critical feedback on the selection form and draft protocol. As the senior author, RS supervised the preparation of the study protocol and addressed the reviewers' comments. All authors have approved this final manuscript.

Funding This work is supported by the Key Laboratory of Evidence-based Medicine and Knowledge Translation of Gansu Province, grant number 20170203. Competing interests None declared.

Patient and public involvement Patients and/or the public were not involved in the design, or conduct, or reporting, or dissemination plans of this research.

Patient consent for publication Not required.

Provenance and peer review Not commissioned; externally peer reviewed.

Open access This is an open access article distributed in accordance with the Creative Commons Attribution Non Commercial (CC BY-NC 4.0) license, which permits others to distribute, remix, adapt, build upon this work non-commercially, and license their derivative works on different terms, provided the original work is properly cited, appropriate credit is given, any changes made indicated, and the use is non-commercial. See: http://creativecommons.org/licenses/by-nc/4.0/.

\section{ORCID iDs}

Xuan Yu http://orcid.org/0000-0002-9390-6009

Yanfang Ma http://orcid.org/0000-0001-8882-2479

Wenbo Meng http://orcid.org/0000-0003-2900-7671

Kehu Yang http://orcid.org/0000-0001-7864-3012

Yaolong Chen http://orcid.org/0000-0002-8598-6504

\section{REFERENCES}

1 Menichetti F, Sganga G. Definition and classification of intraabdominal infections. J Chemother 2009;21 Suppl 1:3-4.

2 Sartelli M, Abu-Zidan FM, Catena F, et al. Global validation of the WSES sepsis severity score for patients with complicated intraabdominal infections: a prospective multicentre study (WISS study). World J Emerg Surg 2015;10:61.

3 Bhangu A, Søreide K, Di Saverio S, et al. Acute appendicitis: modern understanding of pathogenesis, diagnosis, and management. Lancet 2015;386:1278-87

4 Flum DR. Acute Appendicitis - Appendectomy or the "Antibiotics First" Strategy. N Engl J Med 2015;372:1937-43.

5 Zhang J, Zhao C, Chen $\mathrm{H}$, et al. A multicenter epidemiology study on the risk factors and clinical outcomes of nosocomial intra-abdominal infections in China: results from the Chinese antimicrobial resistance surveillance of nosocomial infections (cares) 2007-2016. Infect Drug Resist 2018;11:2311-9.

6 Blot S, Antonelli M, Arvaniti K, et al. Epidemiology of intra-abdomina infection and sepsis in critically ill patients: "AbSeS", a multinational observational cohort study and ESICM Trials Group Project. Intensive Care Med 2019;45:1703-17.

7 Sartelli M, Catena F, Ansaloni L, et al. Complicated intra-abdominal infections worldwide: the definitive data of the CIAOW study. World $J$ Emerg Surg 2014;9:37.

8 Sartelli M, Viale P, Catena F, et al. 2013 WSES guidelines for management of intra-abdominal infections. World J Emerg Surg 2013;8:3.

9 Sartelli M, Chichom-Mefire A, Labricciosa FM, et al. The management of intra-abdominal infections from a global perspective: 2017 WSES guidelines for management of intra-abdominal infections. World J Emerg Surg 2017;12:29.

10 Mazuski JE, Tessier JM, May AK, et al. The surgical infection Society revised guidelines on the management of intra-abdominal infection. Surg Infect 2017;18:1-76.

11 Montravers P, Dupont H, Leone M, et al. Guidelines for management of intra-abdominal infections. Anaesth Crit Care Pain Med 2015;34:117-30
12 Price J. Surgical intervention in cases of general peritonitis. Proc Philadelphia County Med Soc 1905;26:199.

13 Inse I, Evander A, Gustafson I, et al. Influence of peritoneal lavage on objective prognostic signs in acute pancreatitis. Ann Surg 1986;204:122-7.

14 Hartwich JE, Carter RF, Wolfe L, et al. The effects of irrigation on outcomes in cases of perforated appendicitis in children. J Surg Res 2013;180:222-5.

15 Siotos C, Stergios K, Prasath V, et al. Irrigation Versus Suction in Laparoscopic Appendectomy for Complicated Appendicitis: A Metaanalysis. J Surg Res 2019;235:237-43.

16 Hajibandeh S, Hajibandeh S, Kelly A, et al. Irrigation versus suction alone in laparoscopic appendectomy: is dilution the solution to pollution? A systematic review and meta-analysis. Surg Innov 2018;25:174-82.

17 Centre for reviews \& dissemination. Systematic reviews: CRD's guidance for undertaking reviews in health care. New York: University of York, 2009.

18 Shamseer L, Moher D, Clarke M, et al. Preferred reporting items for systematic review and meta-analysis protocols (PRISMA-P) 2015: elaboration and explanation. BMJ 2015;350:g7647.

19 Li L, Tian J, Tian H, et al. Network meta-analyses could be improved by searching more sources and by involving a librarian. J Clin Epidemiol 2014;67:1001-7.

20 Higgins JPT, Altman DG, Gøtzsche PC, et al. The Cochrane collaboration's tool for assessing risk of bias in randomised trials. BMJ 2011;343:d5928.

21 Sterne JA, Hernán MA, Reeves BC, et al. ROBINS-I: a tool for assessing risk of bias in non-randomised studies of interventions. BMJ 2016;355:i4919.

22 Norris SL, Meerpohl JJ, Akl EA, et al. The skills and experience of grade methodologists can be assessed with a simple tool. J Clin Epidemiol 2016;79:150-8.

23 GRADEpro GDT. GRADEpro Guideline Development Tool [Software]. McMaster University, 2015 (developed by Evidence Prime, Inc.), 2015. Available: www.gradepro.org

24 Guyatt GH, Oxman AD, Vist G, et al. GRADE guidelines: 4. Rating the quality of evidence--study limitations (risk of bias). J Clin Epidemiol 2011;64:407-15

25 Guyatt GH, Oxman AD, Montori V, et al. GRADE guidelines: 5. Rating the quality of evidence--publication bias. J Clin Epidemiol 2011;64:1277-82.

26 Guyatt GH, Oxman AD, Kunz R, et al. GRADE guidelines 6. Rating the quality of evidence--imprecision. J Clin Epidemiol 2011;64:1283-93.

27 Guyatt GH, Oxman AD, Kunz R, et al. GRADE guidelines: 7. Rating the quality of evidence--inconsistency. J Clin Epidemiol 2011;64:1294-302.

28 Guyatt GH, Oxman AD, Kunz R, et al. GRADE guidelines: 8. Rating the quality of evidence--indirectness. J Clin Epidemiol 2011;64:1303-10.

29 Guyatt GH, Oxman AD, Sultan S, et al. Grade guidelines: 9. rating up the quality of evidence. J Clin Epidemiol 2011;64:1311-6.

31 StataCorp LLC.Stata: software for statistical and data science. Available: https://www.stata.com/ [Accessed 2020].

31 Higgins JPT, Thomas J, Chandler J. Cochrane Handbook for systematic reviews of interventions. version 6.0. Cochrane, 2019.

32 DerSimonian R, Kacker R. Random-effects model for meta-analysis of clinical trials: an update. Contemp Clin Trials 2007;28:105-14.

33 Peters JL, Sutton AJ, Jones DR, et al. Comparison of two methods to detect publication bias in meta-analysis. JAMA 2006;295:676-80.

34 Maria E, Francois B, Amulya S, et al. Infectious complications after laparoscopic appendectomy in pediatric patients with perforated appendicitis: is there a difference in the outcome using irrigation and suction versus suction only? results of a multicentric international retrospective study. J Laparoendosc Adv Surg Tech A 2018:lap.2018.0061-

35 Nataraja RM, Panabokke G, Chang AD, et al. Does peritoneal lavage influence the rate of complications following pediatric laparoscopic appendicectomy in children with complicated appendicitis? A prospective randomized clinical trial. J Pediatr Surg 2019;54:2524-7.

36 Moher D, Liberati A, Tetzlaff J, et al. Preferred reporting items for systematic reviews and meta-analyses: the PRISMA statement. PLoS Med 2009;6:e1000097.

$37 \mathrm{Ge} \mathrm{L}$, Tian J-H, Li Y-N, et al. Association between prospective registration and overall reporting and methodological quality of systematic reviews: a meta-epidemiological study. J Clin Epidemiol 2018;93:45-55. 